\title{
Competition and Coordination Strategies of Shared Electric Vehicles and Public Transportation Considering Customer Travel Utility
}

\section{Zhiyong Zhang}

Xidian University School of Economics and Management

Xiao Zhang ( $\square$ zhangxiao.neu@163.com)

Xidian University School of Economics and Management https://orcid.org/0000-0001-7174-5657

\section{Research Article}

Keywords: shared electric vehicle, competition and coordination, customer travel utility, public transportation

Posted Date: February 8th, 2021

DOI: https://doi.org/10.21203/rs.3.rs-158987/v1

License: (c) (i) This work is licensed under a Creative Commons Attribution 4.0 International License.

Read Full License

Version of Record: A version of this preprint was published at Environmental Science and Pollution Research on June 29th, 2021. See the published version at https://doi.org/10.1007/s11356-021-15040-9. 


\title{
Competition and coordination strategies of shared electric vehicles and public
}

\section{transportation considering customer travel utility}

\author{
Zhi-yong Zhang, Xiao Zhang * \\ (Department of Management Engineering, School of Economics and Management, Xidian University, Xi'an, China, 710026) \\ (Email: Zhiyong Zhang, 213143719@seu.edu.cn; Xiao Zhang, zhangxiao.neu@163.com)
}

Abstract With the rise of the sharing economy and the concept of "green environmental protection and low-carbon travel", the emerging project of shared electric vehicles is booming. However, the accompanying coordination problem between shared electric vehicles and public transportation system needs to be urgently solved. In reality, customers' choice of travel mode is influenced by their own travel perceived utility. Thus, this paper will discuss the competition and coordination problem between shared electric vehicles and public transportation system from the perspective of customer travel utility. Considering the travel cost and comfort in the customer travel utility, the game models of shared electric vehicle and public transport system in different scenarios are established by using competitive game and cooperative game. Then, the equilibrium solutions under different scenarios are obtained by solving the models. The analysis results show that shared electric vehicles would bring some beneficial improvements to the transportation system under certain circumstances. Furthermore, public transportation system should adopt a coordination strategy with the shared electric vehicles to promote the total customer travel utility for the entire system. It is worth considering the improvement of the service quality of shared electric vehicle and public transportation next, which would affect the rate of increasing in the total customer travel utility.

Keywords shared electric vehicle; competition and coordination; customer travel utility; public transportation

\section{Introduction}

During the past decades, bus, subways and other public transport (PT) as well as private car (PC) have played an important role in urban transportation. In recent years, with the rise of the sharing economy and the concept of "green environmental protection and low-carbon travel", the emerging project of shared electric vehicle (SEV) is developing rapidly, which has caused some changes in people's travel mode. Compared with PT, SEV provides greater comfort and flexibility to travel regardless of route and timetable constraints (Liu et al. 2020). Different from PC, SEV uses more environmentally friendly energy and reduces the waste of social resources (Liu et al. 2020) as well as people's travel costs such as vehicle purchase fee, gasoline, insurance and other related costs (Xu et al. 2020). Recently, an increasing number of people choose SEV to travel. According to statistics from the "In-depth analysis report on China's shared car business model innovation and investment opportunities" released by the Foresight Industry Research 
Institute, the scale of China's SEV market was 1.729 billion yuan in 2017, and it was 3.648 billion in 2018, with a growth rate of $111 \%$. In 2019 , this market reached 6.639 billion yuan. It is predicted that the scale of China's SEV market will exceed 45 billion yuan by 2023 and the average compound growth rate in 2019-2023 is about 61.84\% (In-depth analysis report on China's shared car business model innovation and investment opportunities 2019.). With the rapid development of SEV, the travel modes of customers become diverse, and customers need to choose among these travel modes during travel. In reality, SEV has been already starting to affect PT gradually. On the one hand, SEV attracts customers with its innovative travel methods, travel flexibility and other factors, which is a noteworthy competitor with PT. On the other hand, SEV and PT coordinate to satisfy the diversity of customer travel. For example, customers can use PT to the adjacent SEV parking station and then use SEV to the destination. Thus, for the development of both sides, the coordination problem between SEV and PT needs to be solved urgently, which has gradually attracted the attention of academia and industry.

With the gradual rise and development of SEV, there has been an upsurge of scholarly attention on the research of operational problems of SEV. The majority of the studies focus on the following three problems: rebalancing or scheduling problem (Bruglieri et al. 2014; Weikl and Bogenberger 2015; Li et al. 2016; Correia and Antunes 2017), charging problem (Dong et al. 2014; Avci et al. 2014; Neubauer and Wood 2014; Wu 2019; Paula et al. 2020) and station selection and station planning (He et al. 2017; Kaspi et al. 2016; Mak and Shen 2014; Ang et al. 2012). After years of research on the above problems, it has been found that the impact of other modes of transportation on SEV as well as the coordination problem between them should be considered. Therefore, scholars are increasingly concerned with the coordination problem and the choice between different travel modes in the transportation system (Liu 2012; Zhang et al. 2014; Hao and He 2013; Zhang and Chen 2018; Chen et al. 2016), which can be summarized as the choice and coordination under the competitive conditions (Guo et al. 2000). Research on the coordination problem between SEV and other modes of transportation is rare, but some researches on coordination between other modes of transportation have been paid more attention. In view of such problems, scholars conduct research from two aspects. The first aspect is on the competitive relationship between PC and PT. Yong and Huang (2016) studied customers' choice between subway and driving under different charging strategies. Ci et al. (2017) used lotka-volterra model to describe the evolution of the balance between PT and PC. Liu (2012) put forward the concept of "ecological niche" and pointed out that the "ecological niche" of various travel modes has overlapping competitive relations. Some scholars pointed out that the research in this aspect is not universally applicable, for not all travelers have private transportation (Guo et al. 2000). The second aspect is on the coordination problems among strong accessibility, which refers to buses, subways, taxis, shared bikes, etc. Zhang and Li (2014) set up the utility function to study the competitive relationship 
between the rail transit and buses in the case of collinearity, based on the travel time and cost as the principal variables. Ma et al. (2007) replaced the utility function with the generalized cost function, and used the Logit model to explore the passenger flow sharing rate of the rail transit and buses. Ahmadreza et al. (2017) analyzed the competition of New York City's taxi and bike-sharing system (BSS) in terms of travel time through the data survey of New York City, and believed that BBS system was more time saving in traffic congestion areas. Cantarella and De (2005) adopted the multi-layer feed forward network model to select and predict the mode of transportation. These have informed the research of coordination problems between SEV and other transportation.

Travel utility as well as travel price are often considered by most scholars when examining the above problems. The interest in considering and studying the customer travel utility and preference in the travel choice problem has grown rapidly over the past two decades. Mokhtarian et al. (2001) and Redmond et al. (2001) were the first to describe a new concept in transportation that is the travel liking. Currently, many scholars have demonstrated that individuals might be satisfied in performing the travel and the degree of customer satisfaction with the travel modes would directly affect their choice. Even if relatively new, the travel satisfaction concept shares some common traits with the well-known concept of utility (Sprumont et al. 2017), which is considered as a measure of preference among different alternatives belonging to the same choice set for a decision maker (Ben-Akiva et al. 1985; McFadden 1980). Meanwhile, Whalen et al. (2013) also observed satisfaction feelings during travel. Travel perceived utility is considered as a preference measure that the decision maker chooses between different travel modes. Therefore, a growing number of scholars begin to study travel utility. The results of a travel survey implemented by the University of Luxembourg in 2012 show that the utility function fitted by this survey is positively correlated with the stated commuting satisfaction. The number of publications that provide empirical research on determinants of travel perceived utility is growing rapidly. St-Louis et al. (2014) made an interesting classification between the travel perceived utility determinants, where the internal factors and external factors are opposed. Internal or non-mode specific factors are related to individual characteristics, such as an individual's lifestyle, preference, attitude and so on. External factors or mode-specific attributes are related to travel time, cost, comfort, availability etc. At the same time, the selected travel mode has an important effect on the travel utility. Páez and Whalen (2010) found that the mode of travel with the highest utility for travelers is by bicycle or walking, the mode with medium utility is driving or taking taxis, and the mode of lowest utility is by bus or subway. Some scholars also mentioned that the low utility reported by PT users may be related to the frustration of feeling crowded and uncomfortable (St-Louis et al. 2014; Páez and Whalen 2010).

Previous studies have significantly advanced the research on operational problems of SEV from different perspectives and provided references for this paper. However, there are still some gaps in the 
existing research as follows. (1) Most of the existing studies only consider the competition relationship between different transportation modes, but seldom consider the coordination relationship between them. It is not sound because there is not only competition, but also coordination between different transportation modes in reality. For example, a customer could choose from the following three ways to travel: the first way is to take the bus, the second is to drive a SEV, and the third is to take the bus to an adjacent SEV station and then transfer a SEV to the destination. Thus, on such a journey, the relationship between the bus and SEV is both competition and coordination. This paper will consider the transfer behavior of customers to research the coordination problem between PT and SEV, which is more in line with the reality. (2) Most of the existing research considers the price equilibrium or time equilibrium resolved by converting travel time into travel costs, while the customer's choice is also affected by other factors such as the space comfort besides the price or time cost. In real life, customers often show a dislike to the travel mode that is crowded. Therefore, this paper will introduce the customer travel comfort by calculating the degree of space congestion of travel mode. For example, as the number of customers in the bus increases, the perceived comfort of customer decreases, which could further affect the choice of travel mode. Based on the above analysis, it is essential to study the coordination and competition between SEV and PT from the perspective of customer travel utility, which is consisting of travel comfort and price. This is the motivation of our study.

This paper aims to solve the competition and coordination problem between SEV and PT. The transport system is considered as a closed system that the number of vehicles and customers is constant for the convenience of analysis and calculation. First, the competition and coordination problem between SEV and PT is analyzed through considering the transfer behavior of customers between different travel modes. The equilibrium state and interacting relationship between SEV and PT could be obtained through comparing and analyzing the following three scenarios: (a) only PT in transport system, (b) competition between SEV and PT, (c) competition and coordination between SEV and PT. Then, this paper considers the customer travel utility from the perspective of the space comfort. In addition, there is a correlation between the space comfort and travel price, specifically, the travel price affects the number of customers, which affects the space comfort in turn. Therefore, the impact of travel price and customer quantities on the customer travel utility is considering in this paper. Further, digital simulation experiments are conducted to show the validity of the conclusions obtained from three scenarios.

The rest of this paper is organized as follow. Section 2 gives the basic assumptions of this problem and establishes the customer travel utility function. Section 3 proposes the first and second scenarios of this problem, which are only PT and competition between SEV and PT in transport system, respectively. The third scenario of this problem, which is competition and coordination between SEV and PT, is given in section 4. In section 5, digital simulation experiments are conducted. The paper concludes with a summary 
and an outlook in section 6. Part of the proofs of lemmas is given in the Appendix.

\section{Basic assumptions and the travel utility function}

Based on the reality and previous studies, the basic assumptions and the customer travel utility function are given in this part.

\subsection{Basic assumptions}

Assumption 1. The system is a closed system. It means that the number of PT, SEV and customers in the system are constant and they do not increase or decrease as the system flows. Meanwhile, the travel chain is simplified into three parts: the departure station, the transit station and the terminal station.

Assumption 2. The transport system has two subsystems: PT and SEV, among which only buses and subways are considered in PT since they are the two most common ways in the urban transportation.

Assumption 3. The transportation resources in the system are abundant. To be specific, road resources are sufficient, and customers will not encounter traffic congestion during the travel. Meanwhile, the parking space at the destination is adequate, ensuring that SEV entering the destination can park normally.

Assumption 4. In the SEV subsystem, the power and the number of SEV are sufficient. In other words, customers who use the SEV from any stations can successfully reach their destination and each customer demand would not be missed.

Assumption 5. Customers are homogenous. Each customer has the same utility function for the same travel mode.

Assumption 6. When multiple customers use the same SEV at the same time, they are treated as one. For example, if a couple share the same SEV, they will be regarded as one customer.

\subsection{Travel utility function}

Based on the utility theory, the customer perception utility functions of PT and SEV travel modes are established. The utility function is expressed by the customer's perception of the space comfort, which is affected by the number of customers. In addition, the number of customers is affected by the travel price. The relationship between the customer travel utility of taking SEV or PT and the number of customers are shown in Fig. 1, and the customer travel utility functions of SEV and PT are as follows,

$$
\begin{gathered}
U \text { \$ } E V \equiv \begin{cases}k Q_{S}, & 0 \leq Q_{S}<Q^{\prime} \\
k Q^{\prime}, & Q_{S} \geq Q^{\prime}\end{cases} \\
U \text { P }=a Q_{P}-b Q_{P}{ }^{2}, \quad Q_{P} \geq 0
\end{gathered}
$$

where $k, a$ and $b$ are the perceived coefficients of travel comfort which represent the customer's sensitivity to travel comfort, and these coefficients are constants (Cantarella and De 2005; Páez and Whalen 
2010), $k>0, a>0$ and $b>0$. The values of these coefficients need to be determined by fitting the relevant data of the customer's travel utility survey, which includes offline survey results or online polling and commenting, etc. $Q_{S}$ and $Q_{P}$ are the number of customers who travel by SEV and PT in the function expression, respectively. $Q^{\prime}$ is the maximum number of SEV.

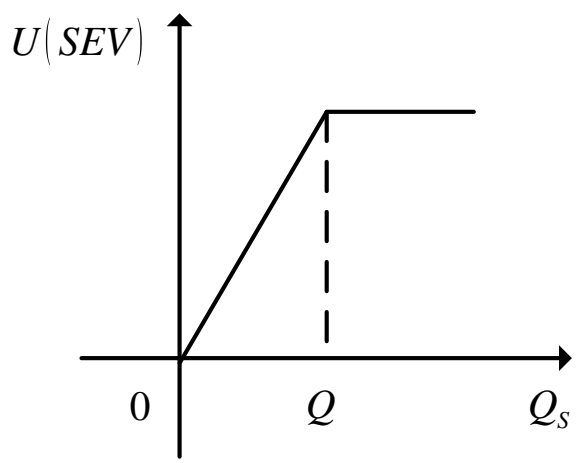

(a) Utility of taking SEV

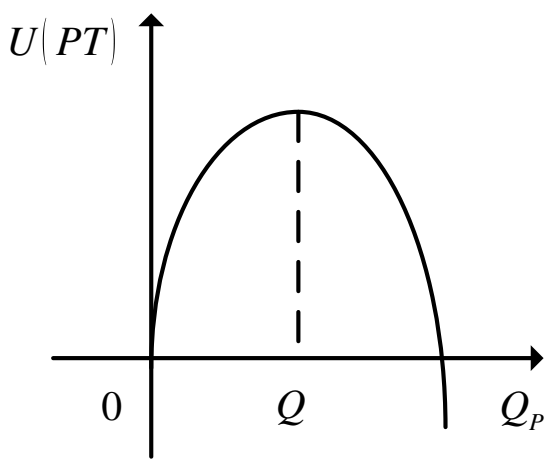

(b) Utility of taking PT

Fig. 1 Travel utility functions of the two subsystem

As shown in Fig. 1, the utility functions of the two subsystems are different. As the number of customer increases, the total travel utility of taking SEV first increases linearly and then remains constant. When customers use SEV, everyone is independent and does not affect each other. According to the Assumption 5, the total travel utility of using SEV is a linear function, which is positive and can be superimposed. Therefore, the first half of total travel utility function of taking SEV increases linearly. Since the number of SEV is finite and appropriate, some customers could not take SEV and the total customer travel utility would not change when the number of customers exceeds the maximum number of SEV. Therefore, the latter half of the total travel utility function is constant. The total travel utility function of taking PT is a quadratic function with an opening downward. When customers use PT for travel, each person is not independent, which means that customers will gradually feel crowded as the number of customers in PT vehicles increases slowly. Therefore, the total travel utility of taking PT gradually increases as the number of customer increases, which is in accordance with the law of diminishing marginal utility. When the number of customers inside a PT vehicle reaches a certain limit, $Q^{\prime \prime}$, customers feel crowded, the travel utility of each customer would become negative and the total travel utility would begin to decline. Even as the number of customers reaches a higher limit, the customers become intolerable and the total travel utility becomes negative.

Customer quantity and the price are not independent of each other. Customer quantity changes in the opposite direction with the travel price, when the price rises, the number of customers decreases (Mokhtarian and Salomon 2001). Therefore, the relationships between travel price and the number of 
customers conform to the Demand Theory (Mokhtarian and Salomon 2001; Zhang and Chen 2018), which are shown in Fig. 2. In addition, the travel demand functions of SEV and PT are as follows.

$$
\begin{aligned}
& Q_{S}=\beta p^{\prime}-c p_{S}, \\
& Q_{P}=Q_{\text {all }}-Q_{S}=Q_{\text {all }}-\beta p^{\prime}+c p_{S},
\end{aligned}
$$

where $Q_{a l l}$ is the total number of customers, which is constant according to Assumption 1. $p^{\prime}$ and $p_{S}$ are the price of PT and SEV in the whole journey, respectively. Based on Assumption 1, it can be known that the travel chain is settled and the travel price of using PT, $p_{P}=p^{\prime}$, is a determined value. In addition, the travel price of taking SEV, $p_{S}$, is a variable. The price of taking PT is the lowest bound on that of SEV since the vicious competition with PT is not considered. Meanwhile, when $p_{S}$ increases, the number of customers, $Q_{S}$, would decrease, so it's a decreasing function (Mokhtarian and Salomon 2001). Besides, $\beta$ and $c$ are the coefficients of price elasticity of demand and these coefficients are constants (Mokhtarian and Salomon 2001; Zhang and Chen 2018), $\beta>0$ and $c>0$. The value of coefficients needs to be obtained by fitting the relevant data of the customer's travel records.

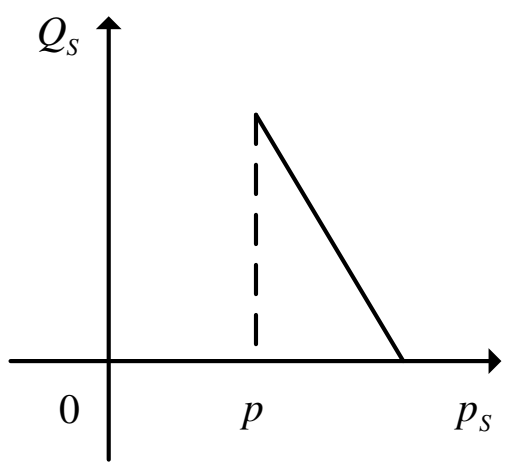

(c) Demand of SEV

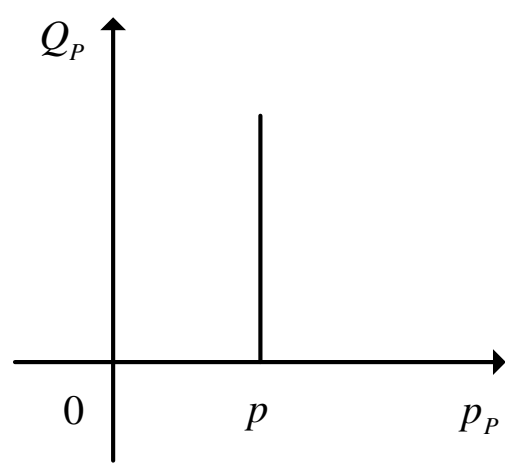

(d) Demand of PT

Fig. 2 Demand functions of the two subsystem

\section{The competitive game between PT and SEV}

In this section, there is no coordination relationship between PT and SEV, but only a competitive relationship exists. In other words, customers in the system use either SEV or PT to travel. To better analyze the impact of SEV on PT under competitive conditions, two different scenarios are considered. The first scenario is only PT in transport system and the second is competition between SEV and PT.

\subsection{Scenario 1: No SEV}

In this scenario, customers can only use PT to travel. The travel process of customers is shown in Fig. 3, where station $\mathrm{A}$ is the departure station and station $\mathrm{C}$ is the terminal station. It should be pointed out that 
customers may ride bicycles or walk to neighboring stations to take PT due to exercise or avoiding overcrowding in reality. Since the distance is close, the customer travel utility and demand functions do not change significantly, this case is treated as the same with that only taking PT.

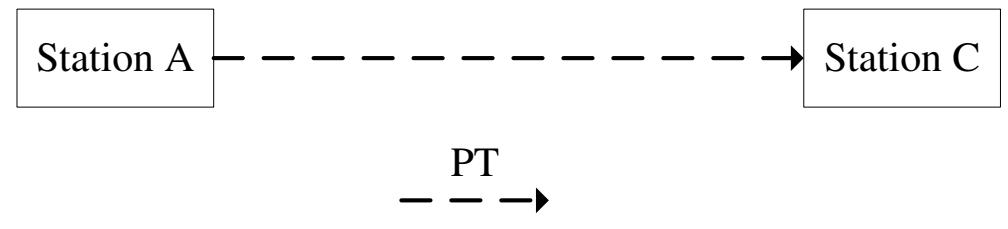

Fig. 3 Customer's travel process for Scenario 1.

The total number of customers, $Q_{a l l}$, is a determined value in the transport system. Owing to only PT in the transport system, customers have no choice but to use PT from station A to station C. In this scenario, $Q_{P}=Q_{a l l}$. Therefore, the total customer travel utility, $U \boldsymbol{Q}_{\text {all }}^{-}$, is as follow.

$$
U \boldsymbol{Q}_{\text {all }}=U \boldsymbol{P} T \bar{\equiv}=a Q_{\text {all }}-b Q_{\text {all }}{ }^{2} .
$$

Taking the derivative of the above expression, we can get $\frac{d U \boldsymbol{Q}_{\text {all }}}{d Q_{\text {all }}}=a-2 b Q_{\text {all }}$. Let $\frac{d U \boldsymbol{Q}_{\text {all }}}{d Q_{\text {all }}}=0$, then we can get $Q_{a l l}=\frac{a}{2 b}$. Therefore, in Scenario 1, the optimal number of customers using PT is $Q_{a l l}{ }^{*}=\frac{a}{2 b}$ and the maximum total utility of the system is $U \boldsymbol{Q}_{\text {all }}{ }^{*}=\frac{a^{2}}{4 b}$.

\subsection{Scenario 2: Competition between PT and SEV}

The travel process of customers in this scenario is shown in Fig. 4. Since there are PT and SEV in the transport system, customers can choose either PT or SEV from station A to station C. The number of customers who travel by PT and SEV are $Q_{P}$ and $Q_{S}=Q_{a l l}-Q_{P}$, respectively. Meanwhile, the price of taking SEV is $p=p_{S}$. In addition, the customer travel utility and demand function of PT or SEV are Eqs. (1)-(4). Based on the above analysis, the total customer travel utility in scenario $2, U \boldsymbol{Q}_{P}, Q_{s}^{-}$, is as follow.

$$
U \boldsymbol{Q}_{P}, Q_{S} \doteq a Q_{P}-b Q_{P}^{2}+k Q_{S}=a Q_{P}-b Q_{P}^{2}+k \boldsymbol{Q}_{\text {all }}-Q_{P} \overline{2}
$$

where $Q_{P}=\beta p^{\prime}-c p$ and $Q_{S}=Q_{a l l}-Q_{P}=Q_{a l l}-\beta p^{\prime}+c p$. 


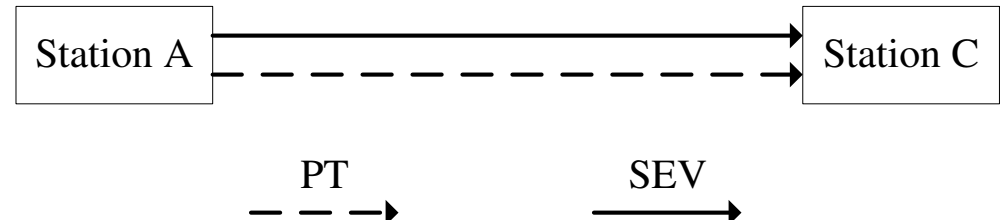

Fig. 4 Customer travel process for Scenario 2.

Taking the derivative of the above expressions, we can get $\frac{d U \boldsymbol{Q}_{P}, Q_{S}}{d Q_{P}}=a-2 b Q_{P}-k$ and $\frac{d U \boldsymbol{Q}_{P}, Q_{S}}{d p}=a c-2 b c \boldsymbol{Q}_{P}-\alpha p^{\prime}+c p=k c$. Let $\frac{d U \boldsymbol{Q}_{P}, Q_{S}}{d Q_{P}}=0$ and $\frac{d U \boldsymbol{Q}_{P}, Q_{S}}{d p}=0$, then we can get the corresponding extreme value point that $Q_{P}=\frac{a-k}{2 b}$ and $p=\frac{a-k-2 b Q_{P}+2 b \beta p^{\prime}}{2 b c}$. Owing to $Q_{S}=Q_{a l l}-Q_{P}$, it can be obtained $Q_{S}=Q_{a l l}-Q_{P}=Q_{a l l}-\frac{a-k}{2 b}$. Calculating the Hessian matrix, it can be obtained that the matrix is a negative definite matrix. Therefore, it can be known that the optimal numbers of customers using $\mathrm{PT}$ and $\mathrm{SEV}$ are $Q_{P}^{*}=\frac{a-k}{2 b}$ and $Q_{S}^{*}=Q_{a l l}-\frac{a-k}{2 b}$, respectively. In this scenario, the maximum total utility of the system is $U \boldsymbol{Q}_{P}^{*}, Q_{S}^{*}=\frac{a_{-k}^{2}}{4 b}+k Q_{S}=\frac{a^{2}}{4 b}+\frac{k+4 b Q_{a l l}-2 a^{-}}{4 b}$.

Compared with Scenario 1, we can summarize the following lemmas and part of proofs are given in Appendix.

Lemma 1. When SEV enters the transport system and does compete with PT, the optimal number of customers using PT is reduced.

Proof. Comparing the optimal number of customers using PT in Scenario 2 with Scenario 1, it can be found that $\frac{a-k}{2 b}<\frac{a}{2 b}$ owing to $a>0, b>0$ and $k>0$. And the number of customers reduced is $\Delta Q_{P}=\frac{k}{2 b}$

Lemma 2. When the total customer quantity overs a certain level, $Q_{a l l}>\frac{a}{2 b}-\frac{k}{4 b}$, the total customer travel utility in the only competition scenario is higher than that when only PT is present in the system. And vice versa. 
Proof. The change in the total utility is $\Delta U=U \mathbf{Q}_{P}^{*}, Q_{S}^{*}=U \mathbf{Q}_{P}^{*}=\frac{k+4 b Q_{a l l}-2 a^{-}}{4 b}$. When $k+4 b Q_{a l l}-2 a>0$, it can be obtained $\Delta U>0$. Therefore, when $Q_{\text {all }}>\frac{a}{2 b}-\frac{k}{4 b}, U \boldsymbol{Q}_{P}^{*}, Q_{S}^{*}>U \boldsymbol{Q}_{P}^{*}{ }^{*}$. In other words, after SEV enters the system and only competes with PT, the total customer travel utility

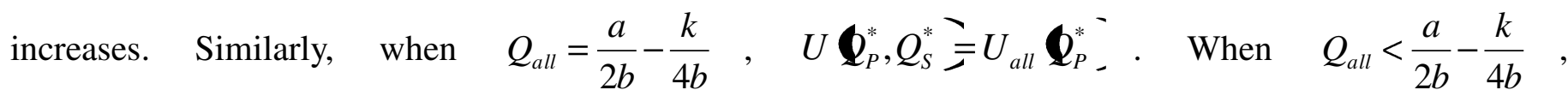
$U \boldsymbol{Q}_{P}^{*}, Q_{S}^{*}<U_{\text {all }} \boldsymbol{Q}_{P}^{*}$; In summary, the magnitude of the total utility of Scenario 1 and Scenario 2 depends on the relationship between $Q_{a l l}$ and $\frac{a}{2 b}-\frac{k}{4 b}$ in the transport system.

Lemma 3. As the number of customers using SEV does not reach a certain level, SEV should be subsidized to promote the total customer travel utility of the whole system. And on the contrary, the result is reversed.

The proof of this lemma is shown in Appendix.

\section{The cooperative game between PT and SEV}

In Scenario 3, PT and SEV have both competition and coordination relationship, which means customers in the transport system can not only use PT but also SEV to travel. Transfer behavior of customers is considered in this scenario by selecting a station, station B, as the transfer station. There are three travel routes for customers, which are shown in Fig. 5. Customers could take PT or SEV from station A to station C directly. Moreover, customers could take PT to station B and then transfer to take SEV to station C. In reality, most customers tend not to change their travel modes after driving or taking a car like SEV or taxi (St-Louis et al. 2014; Páez and Whalen 2010), which is not considered in this paper.

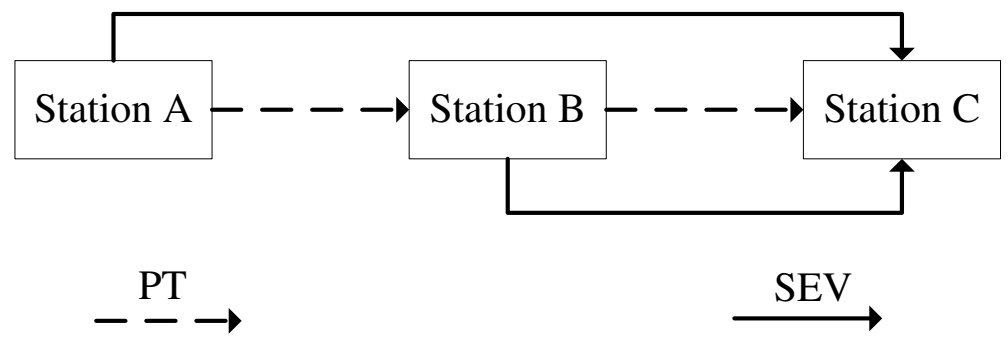

Fig. 5 Customer travel process for Scenario 3.

Since there is a certain distance between station A and station B, the customer travel utility from station A or station B are significantly different, which cannot be ignored at this point. The demand function and customer travel utility of taking SEV to station C directly are expressed as follow.

$$
Q_{S}=\beta p^{\prime}-c p_{A},
$$


where $p_{A}$ is the price of taking SEV from station A, $Q_{S}$ is the number of customer who takes $\mathrm{SEV}$ from station A, $\eta$ is the proportionality coefficient influenced by the distance between station A and station B and the service quality of SEV (Zhang and Li 2014; Ma et al. 2007; Páez and Whalen 2010), $0<\eta \leq 1$. The customer travel utility of taking SEV from station B to C is $\eta$ times as much as that from station A to B, thus the customer travel utility of taking SEV to station C directly can be regarded as the sum of these two parts.

For customers who taking PT at station A then transfer SEV at station B, their travel utility is the sum of the utility of taking PT from station A to B and that of taking SEV from station B to C. Therefore, the demand function and customer travel utility of taking PT first and then transfer SEV to station C are expressed as follow.

$$
\begin{aligned}
& Q_{S}^{\prime}=\beta p^{\prime}-c p_{B}, \\
& Q_{P}=Q_{a l l}-Q_{S}=Q_{a l l}-\beta p^{\prime}+c p_{A}, \\
& U \text { P } t S=a Q_{P}-b Q_{P}^{2}+\eta k Q_{S}^{\prime},
\end{aligned}
$$

where $Q_{P}$ and $Q_{S}^{\prime}$ are the number of customers who take PT from station A and take SEV from station B, respectively. $p_{B}$ is the price of taking SEV from station B. $U \boldsymbol{P}_{t}^{-}$, is the customer travel utility of this travel route.

Since the transfer behavior of customers is considered, some customers who taking PT from station A will transfer SEV at station B and others continue to take PT to station C. Therefore, the number of customer who still takes PT to station $\mathrm{C}$ is

$$
Q_{P}^{\prime}=Q_{P}-Q_{S}^{\prime}=Q_{a l l}-2 \beta p^{\prime}+c p_{A}+c p_{B},
$$

In addition, the customer travel utility of taking PT to station $\mathrm{C}$ directly is

$$
U \boldsymbol{P} T=(+\mu) Q_{P}^{\prime}-b Q_{P}^{\prime 2}
$$

where the proportionality coefficient, $\mu$, is influenced by the distance between station $\mathrm{A}$ and station $\mathrm{B}$, and the service quality of PT, $0<\mu \leq 1$ (Zhang and Li 2014; Ma et al. 2007; Páez and Whalen 2010). The customer travel utility of taking PT from station B to $\mathrm{C}$ is $\mu$ times as much as that from station $\mathrm{A}$ to $\mathrm{B}$, thus the customer travel utility of taking PT to station C directly can be regarded as the sum of these two parts. 
In summary, customers can choose one of the three routes to travel, so the total customer travel utility

Taking the derivative of Eq. (14), we can get $\frac{\partial U_{\text {all }} \boldsymbol{Q}_{P}, Q_{S}, Q_{P}^{\prime}, Q_{S}^{\prime}}{\partial p_{A}}=\mathbf{C}+\mu \mathrm{X}-\mathbf{4}+\eta \mathrm{k} c$, $\frac{\partial U_{a l l} \boldsymbol{Q}_{P}, Q_{S}, Q_{P}^{\prime}, Q_{S}^{\prime}}{\partial p_{B}}=\mathbf{Q}+\mu X-\eta k c-a c+2 b c \boldsymbol{\beta} p^{\prime}-c p_{B_{-}-}, \quad \frac{\partial U_{a l l} \boldsymbol{Q}_{P}, Q_{S}, Q_{P}^{\prime}, Q_{S}^{\prime}=}{\partial Q_{P}^{\prime}}=a-k-2 b \boldsymbol{Q}_{P}-Q_{P}^{\prime}-$ and $\frac{\partial U_{a l l} \boldsymbol{Q}_{P}, Q_{S}, Q_{P}^{\prime}, Q_{S}^{\prime}}{\partial Q_{P}}=\mu a-\eta k+2 b Q_{P}-2 b \boldsymbol{M}+2 \mathcal{Q}_{P}^{\prime}$, where $X=a c-2 b c \boldsymbol{Q}-2 \beta p^{\prime}+c p_{A}+c p_{B}$ - Let

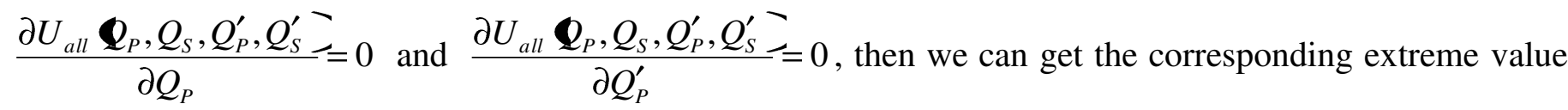
points that $Q_{P}=\frac{a}{b}-\frac{k}{2 b}-\frac{k+\eta_{-}^{\prime}}{2 b+\mu_{-}}$and $Q_{P}^{\prime}=\frac{a}{2 b}-\frac{k+\eta_{-}}{2 b+\mu_{-}}$. Owing to $Q_{S}=Q_{a l l}-Q_{P} \quad$ and $Q_{S}^{\prime}=Q_{P}-Q_{P}^{\prime}, \quad$ it $\quad$ can $\quad$ be obtained $\quad Q_{S}=Q_{a l l}-\frac{a}{b}+\frac{k}{2 b}+\frac{k+\eta}{2 b+\mu_{-}}$and $Q_{S}^{\prime}=\frac{a}{2 b}-\frac{k}{2 b}$. Let

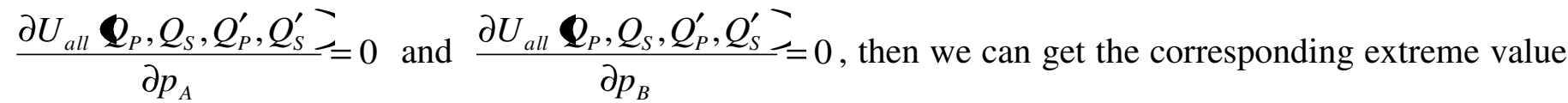
points that $p_{A}=\frac{2 \mathbf{C}+\mu \underline{a}-\mathbf{C}+\mu+\eta \bar{k}-2 b Q_{a l l}+2 b \beta p^{\prime}}{2 b c}$ and $p_{B}=\frac{k-a+2 b \beta p^{\prime}}{2 b c}$. Calculating the Hessian matrix, it can be obtained that the matrix is a negative definite matrix. Therefore, the following conclusions can be obtained from the above results. In Scenario 3, the optimal number of customers taking SEV to station C directly is $Q_{S}^{*}=Q_{S}=Q_{a l l}-\frac{a}{b}+\frac{k}{2 b}+\frac{k+\eta}{2 b\left(+\mu_{-}\right.}$. From station A to station B, the optimal number of customers taking PT is $Q_{P}^{*}=Q_{P}=\frac{a}{b}-\frac{k}{2 b}-\frac{k+\eta}{2 b+\mu_{-}}$and the optimal number of customers transferring $\mathrm{SEV}$ in station B is $Q_{S}^{* *}=Q_{S}^{\prime}=\frac{a}{2 b}-\frac{k}{2 b}$. In addition, the optimal number of customers taking PT to station C directly is $Q_{P}^{\prime *}=Q_{P}^{\prime}=\frac{a}{2 b}-\frac{k+\eta_{-}}{2 b+\mu_{-}}$. In this scenario, the maximum total utility of the system is

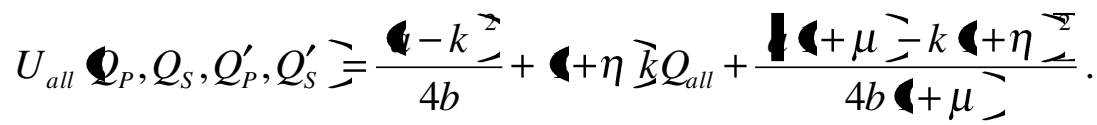
Comparing the equilibrium results of Scenario 2 and Scenario 3 and analyzing the difference between 
them, the lemmas are summarized as follow and part of the proofs is given in Appendix.

Lemma 4. When $\mu<\eta$, the optimal number of customers taking PT in the only competition scenario is higher than that in the competition and coordination scenario, $Q_{P}^{*}>Q_{P}^{*}$, and vice versa.

Proof. As the travel utility function coefficients are determined in Scenario 3, it can be easily found that the size relationship is affected by $u$ and $\eta$. For example, when $\mu<\eta, Q_{P}^{*}-Q_{P}^{* *}=\frac{k-\mu-\mu_{-}^{-}}{2 b}>0$ and $p_{S}-p_{A}=\frac{\mathbf{s}+\mu+\eta \underline{\underline{k}}-\mathbf{C}+2 \mu \underline{\underline{a}}}{2 b c}>0$. Therefore, it could be obtained that the optimal number of customers served by PT is reduced and the optimal price of using SEV at station A is reduced compared to that in Scenario 2. Meanwhile, the amount of change in the optimal number of customers taking PT is $\frac{k-\mu_{1}^{-}}{2 b+\mu_{-}}$. Similarly, when $\mu=\eta, Q_{P}^{*}=Q_{P}^{\prime *}$ and $p_{S}=p_{A}$. When $\mu>\eta, Q_{P}^{*}<Q_{P}^{\prime *}$ and $p_{S}<p_{A}$.

Further conclusions can be drawn from the above. The values of $\mu$ and $\eta$ are influenced by the distance from station A to station B and the service quality of the travel modes (Zhang and Li 2014; Ma et al. 2007; Páez and Whalen 2010). Therefore, when station B in the transport system is settled, the values of $\mu$ and $\eta$ are influenced only by the service quality. As $\mu<\eta$, PT should promptly improve its service quality to get more customers. In addition, the price of taking SEV from station A in Scenario 3 is lower than that in Scenario 2, $p_{A}<p_{S}$, and the reduction is $\frac{\mathbf{c}+2 \mu \underline{q}-\mathbf{c}+\mu+\eta \underline{\underline{k}}}{2 b c}$. Based on the demand function of SEV, when the price of taking SEV decreases, it could result in the increasing number of customer taking SEV and the decreasing number of customer taking PT at station A. It explains why the optimal number of customers traveling by PT decreases in Scenario 3.

Lemma 5. The total customer travel utility in the coordination and competition scenario is higher than that in the only competition scenario, i.e., $U_{\text {all }} \boldsymbol{Q}_{P}, Q_{S}, Q_{P}^{\prime}, Q_{S}^{\prime}>U_{\text {all }} \boldsymbol{Q}_{P}, Q_{S}^{-}$;

Proof. The difference between $U_{\text {all }} \boldsymbol{Q}_{P}, Q_{S}, Q_{P}^{\prime}, Q_{S_{-}^{\prime}}^{\prime-}$ and $U_{\text {all }} \boldsymbol{Q}_{P}, Q_{S_{-}}^{-}$is $\Delta U=U_{\text {all }} \boldsymbol{Q}_{P}, Q_{S}, Q_{P}^{\prime}, Q_{S}^{\prime}$ $-U_{\text {all }} \boldsymbol{Q}_{P}, Q_{S} \equiv \eta k Q_{a l l}+\frac{1+\mu-k \eta_{-}^{\top}}{4 b\left(+\mu_{-}^{\top}\right.}$. Due to the positive parameter values, $\Delta U>0$ and it is always increasing regardless of the size relationship between $\mu$ and $\eta$. The total customer travel utility in the coordination and competition scenario is higher than that in the only competition scenario. Therefore, SEV and PT should adopt a strategy of both competitive and coordination with each other. 
Lemma 6. The variety of total customer travel utility, $\Delta U=U_{\text {all }} \boldsymbol{Q}_{P}, Q_{S}, Q_{P}^{\prime}, Q_{S}^{\prime}=-U_{\text {all }} \boldsymbol{Q}_{P}, Q_{S}^{-}$, is strictly increased as the parameter $\eta$ increases, while it increases or decreases depending on the different value of the parameter $\mu$.

The proof of this lemma is shown in Appendix.

\section{Simulation experiment and analysis}

In order to compare the equilibrium results in different scenarios more clearly and visually, the equilibrium results are summarized in Table 1 below.

Table 1 The equilibrium results of three scenarios.

\begin{tabular}{|c|c|c|c|}
\hline & Scenario 1 & Scenario 2 & Scenario 3 \\
\hline $\begin{array}{l}\text { The optimal number } \\
\text { of customers(PT) }\end{array}$ & $Q_{P}^{*}=\frac{a}{2 b}$ & $Q_{P}^{*}=\frac{a-k}{2 b}$ & $Q_{P}^{\prime *}=\frac{a}{2 b}-\frac{k+\eta_{-}}{2 b(+\mu)}$ \\
\hline $\begin{array}{l}\text { The optimal number } \\
\text { of customers(SEV) }\end{array}$ & - & $Q_{S}^{*}=Q_{a l l}-\frac{a-k}{2 b}$ & $Q_{s}^{*}=Q_{a l l}-\frac{a}{b}+\frac{k}{2 b}+\frac{k(+\eta)}{2 b\left(+\mu_{-}^{-}\right.}$ \\
\hline $\begin{array}{l}\text { The number of } \\
\text { customers(Transfer) }\end{array}$ & - & - & $Q=\frac{a-k}{2 b}$ \\
\hline $\begin{array}{l}\text { The total customer } \\
\text { travel utility }\end{array}$ & $U=\frac{a^{2}}{4 b}$ & $U=\frac{4-k_{2}^{2}}{4 b}+k Q_{\text {all }}$ & 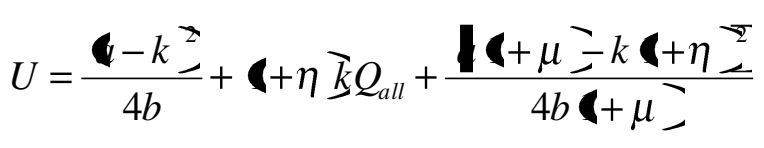 \\
\hline The optimal price & & $a-k-2 b O+2 b \beta p^{\prime}$ & $p_{A}=\frac{2\left(+\mu \underline{a}-\mathbf{C}+\mu+\eta \underline{\underline{k}}-2 b Q_{a l l}+2 b \beta p^{\prime}\right.}{2 b c}$ \\
\hline$(\mathrm{SEV})$ & - & $p_{S}=\frac{a-2 b Q_{\text {all }}+2 v P P}{2 b c}$ & $p_{B}=\frac{k-a+2 b \beta p^{\prime}}{2 b c}$ \\
\hline
\end{tabular}

It can be visualized from Table 1 that equilibrium results are significantly different in three scenarios. And it would bring certain beneficial improvements to the transport system after SEV enters under certain circumstances. In addition, the maximum total customer travel utility can be seen in Scenario 3.Therefore, after SEV enters the transport system, PT should adopt a coordination and competition strategy with it to ensure the maximization of total customer travel utility for the entire system.

Next, numerical simulation experiments are conducted for Scenario3 to further analyze the impact of $\mu$ and $\eta$ on the total customer travel utility. When station B in the transport system is settled, the values of $\mu$ and $\eta$ are influenced only by the service quality of PT and SEV (Zhang and Li 2014; Ma et al. 2007; Páez and Whalen 2010). Two cases are considered based on Lemma 6. In case 1 , set $a=10, b=0.05$, $k=1$ and $Q_{a l l}=200$ and it can be obtained that $\mu<\frac{1}{2 b}+\frac{k+\eta}{a}=1$. Based on Eq. (16), line charts of 
sensitivity analysis under different values of $\mu$ and $\eta$ are shown in Fig. 6.
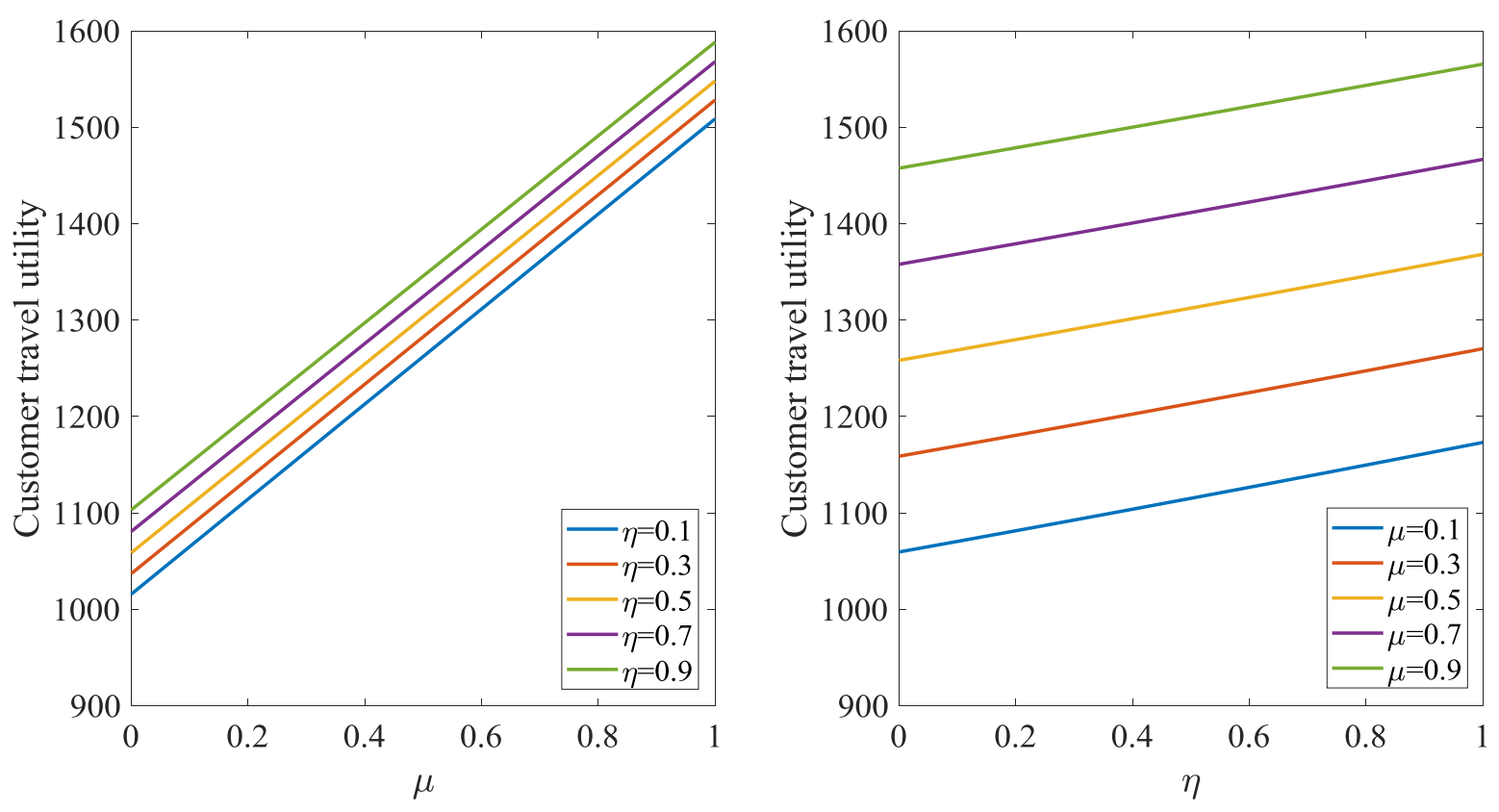

Fig. 6 Sensitivity analysis under different values of $\mu$ and $\eta$.

According to Fig. 6, it could be clearly seen that the total customer travel utility increases at a slower rate as $\eta$ increases when the value of $\mu$ is fixed. Conversely, the total customer travel utility increases at a faster rate with increasing $\mu$ when the value of $\eta$ is fixed. Meanwhile, it can be intuitively reflected from Fig. 6 that different values of $\eta$ have less impact on the change of total customers travel utility than $\mu$. It is consistent with Lemma 6 .

Then, a contrast experiment is set up to research the impact of $\mu$ and $\eta$ on customer travel utility better. In case 2, set $a=10, b=0.6, k=1$ and $Q_{a l l}=200$ in this controlled experiment. This case only changes the value of $b$ so that the value of $\frac{1}{2 b}+\frac{k+\eta}{a}-1$ changes and $\mu>\frac{1}{2 b}+\frac{k+\eta}{a}=1$. Based on Eq. (14), line charts of sensitivity analysis under different values of $\mu$ and $\eta$ are shown in Fig. 7.

It can be clearly seen the difference between Fig. 6 and Fig. 7. The conclusion drawn from Fig. 7 is the opposite of that from Fig. 6. It is consistent with Lemma 6. In other words, these numerical experiments demonstrate the validity of Lemma 6. 

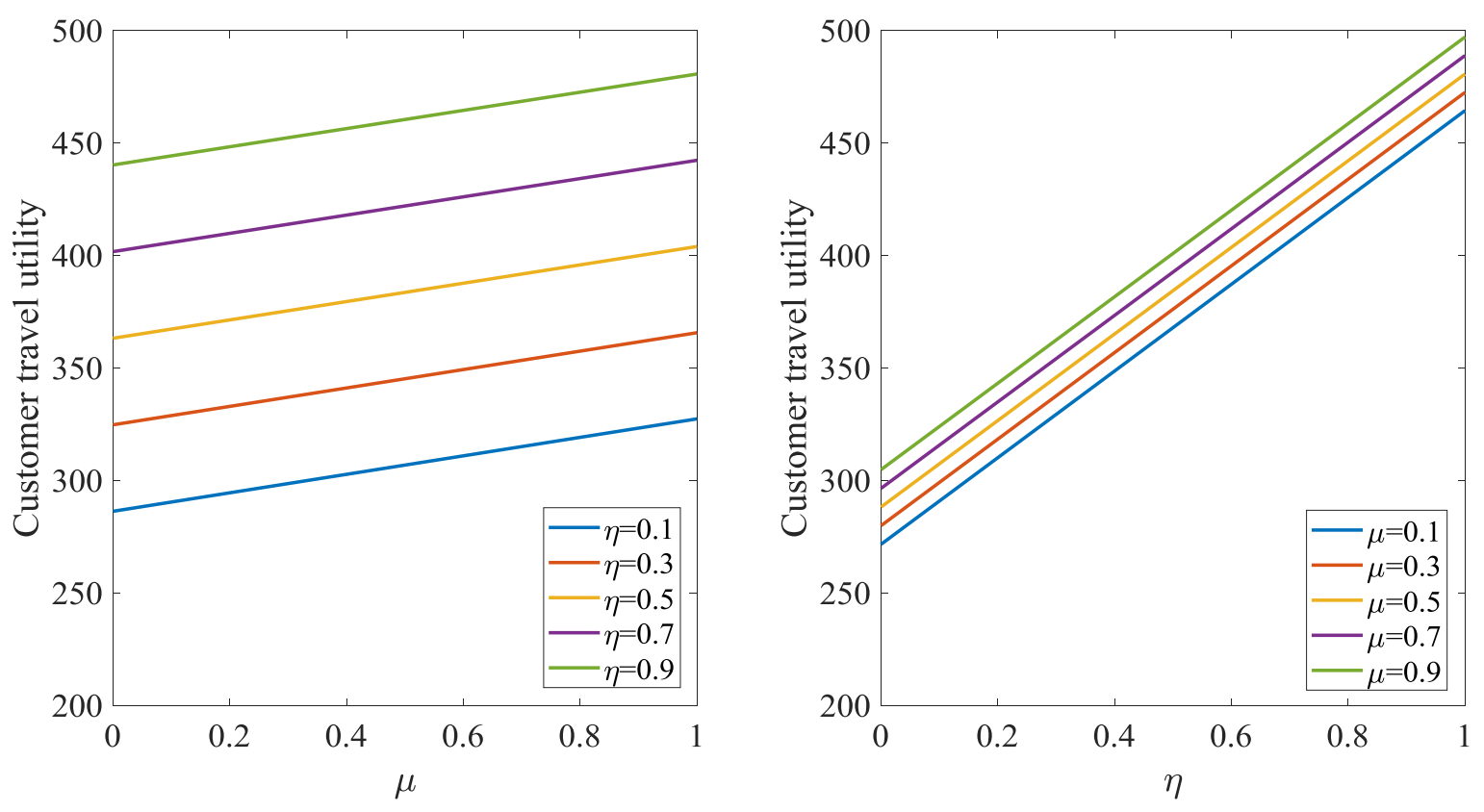

Fig. 7 Contrast experiment under different values of $\mu$ and $\eta$.

Finally, the three-dimensional map is drawn to better display the impact of $\mu$ and $\eta$ on customer travel utility. Based on the Eq. (14), sensitivity analysis under different values of $\mu$ and $\eta$ are shown in

Fig. 8 .

utility increases with increasing $\mu$ at a rapid rate, while the increase of $\eta$ has less effect on the increase

Fig. 8 The impact of $\mu$ and $\eta$ on customer travel utility.

It can be intuitively reflected from Fig. 8 that when $\mu<\frac{1}{2 b}+\frac{k+\eta}{a}-1$, the total customer travel

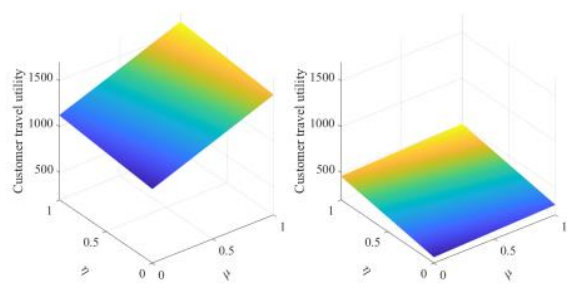
387 in customer travel utility. On the contrary, when $\mu>\frac{1}{2 b}+\frac{k+\eta}{a}-1$, these effects are opposite. Since the 
values of $\mu$ and $\eta$ are influenced only by the service quality of PT and SEV, when $\mu<\frac{1}{2 b}+\frac{k(+\eta}{a}-1$, PT should promptly and emphatically improve its service quality and SEV should maintain or slowly improve its service quality, and vice versa.

\section{Conclusion}

The competition and coordination problem between SEV and PT is solved in this paper by considering the customer travel utility. Considering the travel price and comfort in the customer travel utility, the game models of SEV and PT in three different scenarios are set up and the optimal equilibrium results are obtained. Some conclusions are drawn by comparing and analyzing these optimal equilibrium results and the numerical simulation experiments are conducted to prove the validity of them.

This paper researches competition and coordination problem between SEV and PT by considering the transfer behavior of customers. Based on the reality, the diversity of customer travel makes transport modes no longer a mere competition, which has rarely been considered in the previous research. In addition, this paper considers the customer travel utility from the perspective of the space comfort, which is realistic and enriches study of the travel utility factors. Previous research has rarely considered the impact of space comfort on the customer travel choice, while the customer would resent the crowded travel in reality and change their travel choice as a result. The evolution of the transportation system is investigated in this paper from the initial existence of only PT to the existence of the competition between SEV and PT, and then to the existence of the competition and coordination between SEV and PT. The following conclusions are drawn by comparing and analyzing the equilibrium of different scenarios. First, the total customer travel utility in the competition and coordination scenario is higher than that in the only competition scenario. After SEV enters the transport system, PT should adopt the strategy of competition and coordination with it to maximize the total customer travel utility of the whole system. Meanwhile, after the position of the transit station in the travel chain is determined, it is worth considering the improvement of the service quality of SEV and PT, which affects the increase or decrease of the total customer travel utility. Second, when the number of customers taking SEV does not reach the optimal equilibrium result, the total customer travel utility is less than that in the equilibrium. It is necessary to subsidize SEV at this point to attract more customers and thus ultimately promote the total customer travel utility. Finally, as SEV enters the transport system, the optimal number of customers taking PT in competition scenario is greater than that of in competition and cooperation scenario when the proportionality coefficient of taking PT is lower than that of taking SEV. As the transfer station is settled, the proportionality coefficient is affected by only the service quality. Therefore, it is wise for PT to promote its service quality to attract more customers. On the contrary, 
it is wise for SEV to promote its service quality to attract more customers.

In future studies, we will consider the coordination problem between SEV and PT under the open travel chain, where customers and SEV is allowed to enter or leave the travel chain. It is worthwhile to consider the three-way coordination problem of SEV, PT and taxi in the future research. Taxi is one of the most common ways of public transportation used by customers, while there are a few difference between Taxi and other public transportation like bus and subway. In addition, the coordination problem between SEV and PT with road congestion will be considered.

\section{Authors' contributions}

Zhiyong Zhang analyzed the research problem, wrote the original draft based on the formal analysis and finished the revision. He was also responsible for the drawing part of the paper and for some calculation with software. Xiao Zhang had provided the methodology support and reviewed and edited the writing, and also funded this manuscript. All authors read and approved the final manuscript.

\section{Funding}

This study is partially supported by the National Natural Science Foundation of China (Program No. 71974154), the Natural Science Foundation of Shaanxi Province (Program No. 2019JM-110) and the Fundamental Research Funds for the Central Universities (Project Nos. JB190604 and RW180173).

\section{Data availability}

Data sharing is not applicable to this article as no dataset were generated or analyzed during the current study.

\section{Compliance with ethical standards}

Ethical approval and consent to participate Not applicable.

Consent for publication Not applicable.

Competing interest The author declare that they have no competing interest.

\section{References}

Ahmadreza F I, Anowar S, Mille E J, et al. Hail a Cab or Ride a Bike? A Travel Time Comparison of Taxi and Bicycle-sharing Systems in New York City. Transportation Research Part A: Policy \& Practice, 2017 (101): 11-21.

Ang M, Lim Y F, Sim M. Robust storage assignment in unit-load warehouses. Management Science. 2012, 58(11): 2114-2130.

Avci B, Girotra K, Netessine S. Electric vehicles with a battery switching station: adoption and environmental impact. Management Science. 2014, 61: 772-794.

Banister D. The sustainable mobility paradigm. Transport Policy, 2008 15(2): 73-80.

Ben-Akiva, Moshe E, Lerman S R. Discrete Choice Analysis: Theory and Application to Travel Demand. MIT Press. 1985: 4-25. 
Bruglieri M, Colorni A, Luè A. The vehicle relocation problem for the one-way electric vehicle sharing: an application to the Milan case. Procedia social behavioral science, 2014, 111: 18-27.

Cantarella G E, De L S. Multilayer Feed forward Networks for Transportation Mode Choice Analysis: An Analysis and a Comparison with Random Utility Models. Transportation Research Part C: Emerging Technologies, 2005, 13 (2): 121-155.

Chen P, Zhang Z, Hu X F. Analysis of Passenger Behaviors Impacting on Subway Station Security Test. Urban Mass Transit, 2016, 19 (5): 5-9.

Chorus C G. Logsums for utility-maximizers and regret-minimizers, and their relation with desirability and satisfaction. Transportation Research Part A: Policy and Practice, 2012 46(7): 1003-1012.

Ci Y S, Zhao J F, Wu L N. Lotka-Volterra- based Competition Mode of Transportation in Urban Passenger Corridor. Journal of Transportation Systems Engineering and Information Technology, 2017, 17 (3): 39-45,52.

Correia G, Antunes A. Optimization approach to depot location and trip selection in one-way car sharing systems. Transport Research Part E, 2017, 48: 233-247.

Dong J, Liu C, Lin Z. Charging infrastructure planning for promoting battery electric vehicles: an activity-based approach using multiday travel data. Transportation Research Part C, 2014, 38: 44-55.

Ettema D, Gärling T, Olsson L E, Friman M, Moerdijk S. The road to happiness: Measuring Dutch car drivers' satisfaction with travel. Transport Policy, 2013, 27: 171-178.

Guo X C, Wang W, Lv S. Study on URT's Forecast Model on Cooperate and Competitive OD Matrix. Journal of Highway and Transportation Research and Development, 2000, 17 (4): 57-59, 63.

Hao Z N, He Y H. Pros and Cons of Regular Traffic and Transportation and Innovative Improvement in New Metro Age. Shanghai Urban Management, 2013, 22 (2): 59-62.

He L, Mak H Y, Rong Y, Shen Z. Service region design for urban electric vehicle sharing systems. Manufacturing and Service Operations Management. 2017, 19: 309-327.

Kaspi M, Raviv T, Tzur M, Galili H. Regulating vehicle sharing systems through parking reservation policies: analysis and performance bounds. European Journal of Operational Research. 2016, 251: 969-987.

Li X, Ma J, Cui J, Ghiasi A, Zhou F, Design framework of large-scale one-way electric vehicle sharing systems: a continuum approximation model. Transport Research Part B, 2016, 88: 21-45.

Liu R, Ding Z H, Jiang X, Sun J, Jiang Y L, Qiang W. How does experience impact the adoption willingness of battery electric vehicles? The role of psychological factors. Environmental Science and Pollution Research. 2020, 27: 25230-25247.

Liu X T. An Analysis on the Competitive Tension of Travel Modes and the Heuristic Thoughts from Ecology. Urban Planning Forum, 2012 (5): 66-75.

Ma C Q, Wang Y P, Chen K M. Competition Model between Urban Rail and Bus Transit. Journal of Transportation Systems Engineering and Information Technology, 2007, 7 (3): 140-143.

Mak H Y, Shen Z-JM. Pooling and dependence of demand and yield in multiple-location inventory systems. Manufacturing and Service Operations Management. 2014, 16(2): 263-269.

McFadden D. Econometric Models for Probabilistic Choice Among Products. The Journal of Business. 1980, 53(3): 13-29.

Mokhtarian P L, Salomon I. How derived is the demand for travel? Some conceptual and measurement considerations. Transportation Research Part A: Policy and Practice, 2001 35(8), 695-719.

Neubauer J, Wood E. The impact of range anxiety and home, workplace, and public charging infrastructure on simulated battery electric vehicle lifetime utility. Power Sources. 2014, 257:12-20.

Páez A, Whalen K. Enjoyment of commute: A comparison of different transportation modes. Transportation Research Part A: Policy and Practice, 2010, 44(7): 537-549.

Paula B M, Elías H P, Ángel P N, David AS. Light electric vehicle charging strategy for low impact on the grid. Environmental Science and Pollution Research. 2020, https://doi.org/10.1007/s11356-020-08901-2.

Redmond L S, Mokhtarian P L. The positive utility of the commute: modeling ideal commute time and relative desired commute amount. Transportation, 2001, 28(2): 179-205.

Russell M, Mokhtarian P. How real is a reported desire to travel for its own sake? Exploring the 'teleportation' concept in travel behaviour research. Transportation, 2014, 42(2): 333-345. 
Sprumont F, Paola A, Francesco V. Analyzing the correlation between commuting satisfaction and travelling utility. Transportation Research Procedia. 2017, 25: 2639-2648.

Sprumont F, Viti F, Caruso G, König A. Workplace Relocation and Mobility Changes in a Transnational Metropolitan Area: The Case of the University of Luxembourg. Transportation Research Procedia, 2014, 4: 286-299.

St-Louis E, Manaugh K, Lierop D, El-Geneidy A. The happy commuter: A comparison of commuter satisfaction across modes. Transportation Research Part F: Traffic Psychology and Behaviour, 2014, 26, Part A: 160-170.

The Foresight Industry Research Institute. In-depth analysis report on China's shared car business model innovation and investment opportunities. https://bg.qianzhan.com/trends/detail/506/190517-5da8cc05.html. Accessed May 2019.

Weikl S, Bogenberger K, A practice-ready relocation model for free-floating carsharing systems with electric vehicles-mesoscopic approach and field trial results. Transport Research Part C, 2015, 57: 206-223.

Whalen K E, Páez A, Carrasco J A. Mode choice of university students commuting to school and the role of active travel. Journal of Transport Geography, 2013, 31: 132-142.

Wu P. Which battery-charging technology and insurance contract is preferred in the electric vehicle sharing business? Transportation Research Part A: Policy and Practice, 2019, 124: 537-548.

Xu G W, Wang S Y, Li J, Zhao D T. Moving towards sustainable purchase behavior: examining the determinants of consumers' intentions to adopt electric vehicles. Environmental Science and Pollution Research. 2020, 27: 22535-22546.

Yong G, Huang H J. Modal Split in a Competitive System of Transit and Highway with Heterogeneous Users. Systems Engineering-Theory And Practice, 2016, 36 (9): 2320-2327.

Zhang F, Chen K. Transportation Cost, Scale Effect and Regional Economic Disparity: Taking Liaoning as an Example. Journal of Northeastern University: Natural Science Edition, 2018, 39 (2): 293-296.

Zhang J L, Li T Z. Urban Rail Transit and Bus Collinear Analysis Based on Competition Model. Journal of Transport Information and Safety, 2014, 32 (4): 108-112,118.

Zhang Y, Liu X M, Zhang H. Dilemmas and Strategies for the Development of Urban Non-notorized Travel. Urban Development Studies, 2014, 21 (6): 113-116.

\section{Appendix}

\section{Proof Lemma 3.}

In Scenario 2, it can be known that the optimal numbers of customers using PT and SEV are $Q_{P}^{*}=\frac{a-k}{2 b}$ and $Q_{S}^{*}=Q_{a l l}-\frac{a-k}{2 b}$, respectively. In addition, the maximum total utility of the system is $U \boldsymbol{Q}_{P}^{*}, Q_{S}^{*}=\frac{a^{2}}{4 b}+\frac{k+4 b Q_{a l l}-2 a^{-}}{4 b}$

Since $Q_{\text {all }}$ is a determined value in the transport system, when the number of customers taking SEV does not reach the equilibrium result, i.e., $Q_{S}<Q_{a l l}-\frac{a-k}{2 b}$, it could be obtained that $Q_{P}>\frac{a-k}{2 b}$.In this case, the total utility is less than that in the equilibrium, $U \boldsymbol{Q}_{P}, Q_{S} \leq U \boldsymbol{Q}_{P}^{*}, Q_{S}^{*}=\frac{a^{2}}{4 b}+\frac{k+4 b Q_{a l l}-2 a_{-}^{-}}{4 b}$. Thus, SEV needs to be subsidized to lower the price of taking SEV to attract more customers and ultimately promote the total customer travel utility of the whole system. Similarly, when $Q_{S}=Q_{a l l}-\frac{a-k}{2 b}$, it could be 
obtained $Q_{P}=\frac{a-k}{2 b}$ and $U \boldsymbol{Q}_{P}, Q_{S}=U \boldsymbol{Q}_{P}^{*}, Q_{S}^{*}{ }_{-}^{-}$SEV does not need to be subsidized to upset the optimal equilibrium of the whole system. When $Q_{S}>Q_{a l l}-\frac{a-k}{2 b}$, it can have $Q_{P}<\frac{a-k}{2 b}$ and $U \boldsymbol{Q}_{P}, Q_{S}{ }^{-}<U \boldsymbol{Q}_{P}^{*}, Q_{S}^{*}$. Therefore, SEV does not need to be subsidized, but PT needs to be subsidized to improve the service quality to attract more customers and ultimately promote the total customer travel utility of the whole system.

In summary, the total customer travel utility of the system varies with the relationship between $Q_{S}$

and $Q_{\text {all }}-\frac{a-k}{2 b}$. When $Q_{S}<Q_{\text {all }}-\frac{a-k}{2 b}$, the total customer travel utility of the whole system is decreasing. Therefore, it is recommended to subsidize SEV to promote the total customer travel utility of the whole system in this case. On the contrary, the result is reversed.

\section{Proof Lemma 6.}

Taking the derivations of $\Delta U$, it can be obtained that $\frac{\partial \Delta U}{\partial \eta}=k\left[Q-\frac{a}{2 b}+\frac{k\left(+\eta_{-}^{-}\right.}{2 b(+\mu}\right]=k Q_{-}^{-}$and

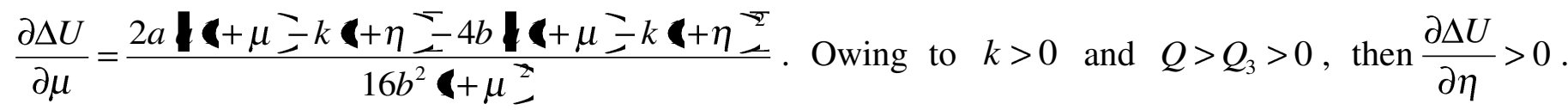
Therefore, $\Delta U$ is strictly monotonically increasing as the value of $\eta$ increases. Let $\frac{\partial \Delta U}{\partial \mu}=0$, we can get

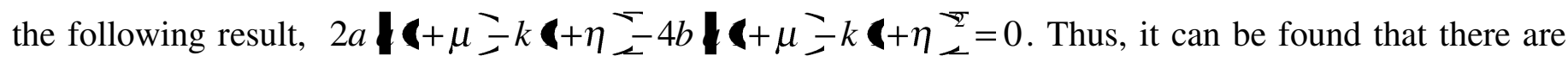
two different cases between $\mu$ and $\frac{k\left(+\eta^{-}\right.}{a}=1$ as follow.

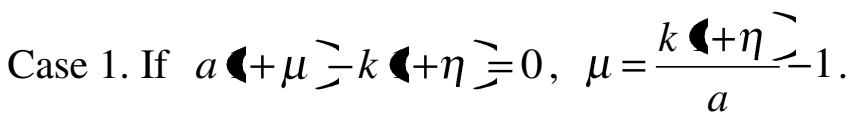

Since $\mu=\frac{k+\eta}{a}=1$, it can be obtained that $\frac{\partial \Delta U}{\partial \mu} \equiv 0$. Therefore, $\Delta U$ is not changed as the value of $\mu$ changes. At this time $\Delta U$ is a constant value, and this constant value is $\Delta U=\eta k Q$.

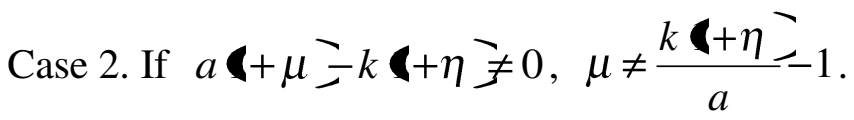

Let $\frac{\partial \Delta U}{\partial \mu}=0$, we can get $\mu=\frac{1}{2 b}+\frac{k+\eta}{a}-1$, and the following conclusions can be drawn. When 
$558 \mu>\frac{1}{2 b}+\frac{k+\eta}{a}=1, \frac{\partial \Delta U}{\partial \mu}<0, \Delta U$ decreases as the value of $\mu$ increases at this time. When $\mu<\frac{1}{2 b}+\frac{k+\eta}{a}-1, \frac{\partial \Delta U}{\partial \mu}>0, \Delta U$ increases as the value of $\mu$ increases at this time. Thus, $\mu=\frac{1}{2 b}+\frac{k+\eta}{a}=1$ is the only maximum point of $\Delta U$. Therefore, $\mu=\frac{1}{2 b}+\frac{k+\eta}{a}=1$ is the maximum point of $\Delta U$.

For $\mu>0$, we need to discuss the above calculations by different situations.

(1) When $\mu=\frac{1}{2 b}+\frac{k+\eta}{a}=1 \leq 0$, it means that $\mu=0$ is the only maximum point of $\Delta U$, and $\Delta U_{\max }<\eta k Q+\frac{\boldsymbol{1}-k+\eta_{\mathbf{T}}^{\mp}}{4 b} . \Delta U$ increases as the value of $\mu$ increases at this time.

(2) When $\mu=\frac{1}{2 b}+\frac{k\left(+\eta^{-}\right.}{a}=1>0, \Delta U$ decreases as the value of $\mu$ increases. It means that

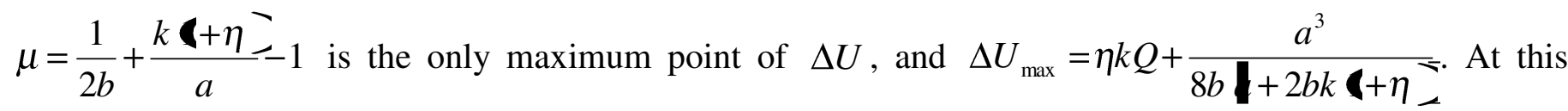
time, when $0<\mu<\frac{1}{2 b}+\frac{k+\eta}{a}=1, \Delta U$ increases as the value of $\mu$ increases. Thus, it means that $\mu=\frac{1}{2 b}+\frac{k(+\eta)}{a}=1$ is the only maximum point of $\Delta U$, and $\Delta U_{\max }=\eta k Q+\frac{a^{3}}{8 b+2 b k+\eta_{-}}$.

Therefore, the increase or decrease of $\Delta U$ is different depending on the value of $\mu$. There are three different relationships between $\Delta U$ and $\mu$. When $\mu=\frac{k+\eta^{-}}{a}-1, \Delta U$ is a fixed value and the fixed value is $\eta k Q$. When $\mu \neq \frac{k(+\eta)}{a}=1, \mu=\frac{1}{2 b}+\frac{k(+\eta)}{a}=1$ is the only maximum point of $\Delta U$. If $\frac{1}{2 b}+\frac{k(+\eta}{a}-1 \leq 0 \quad, \quad \Delta U \quad$ is strictly increased as $\mu$ increases at this time and

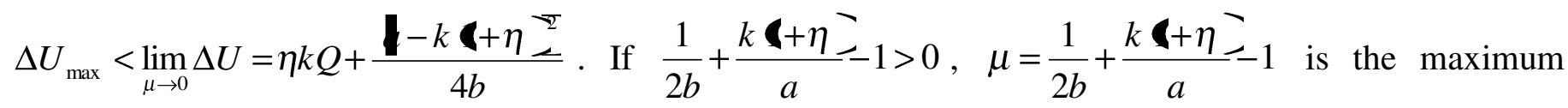
point of $\Delta U$ at this time and $\Delta U_{\max }=\eta k Q+\frac{a^{3}}{8 b+2 b k+\eta_{-}}$. 
Figures

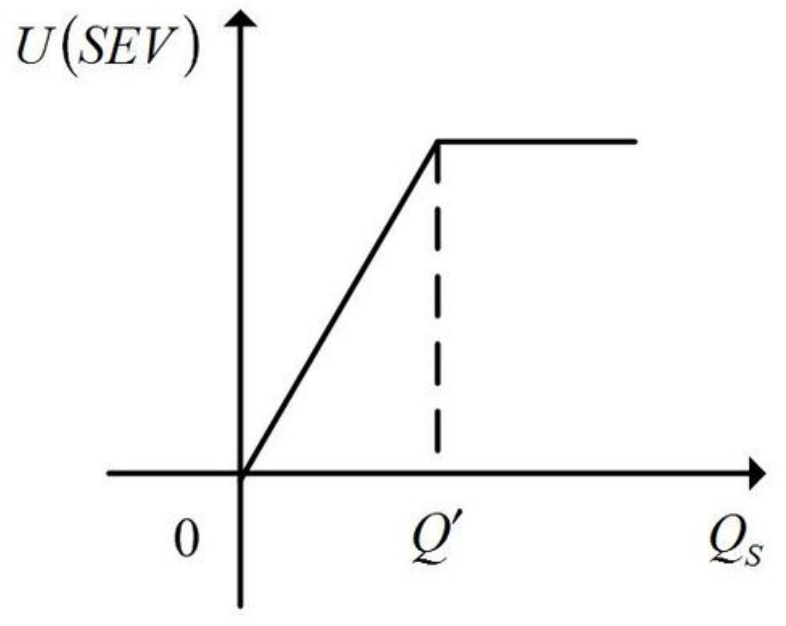

(a) Utility of taking SEV

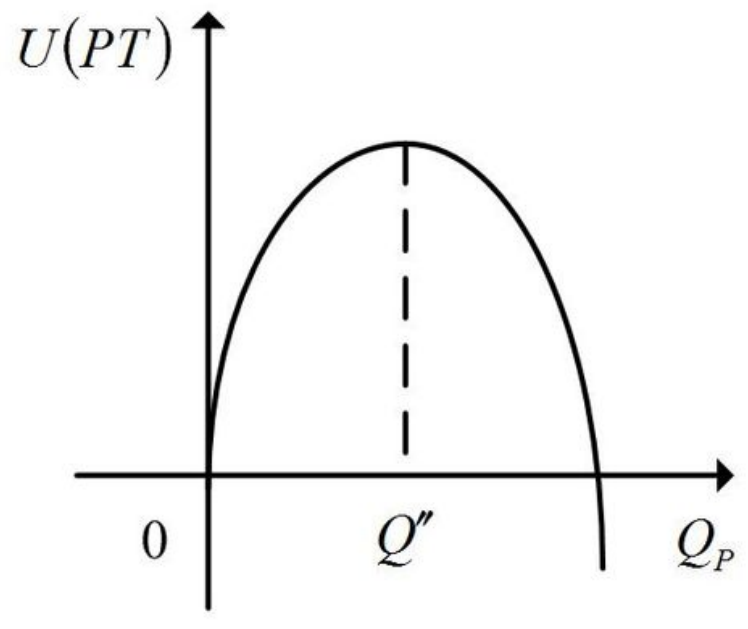

(b) Utility of taking PT

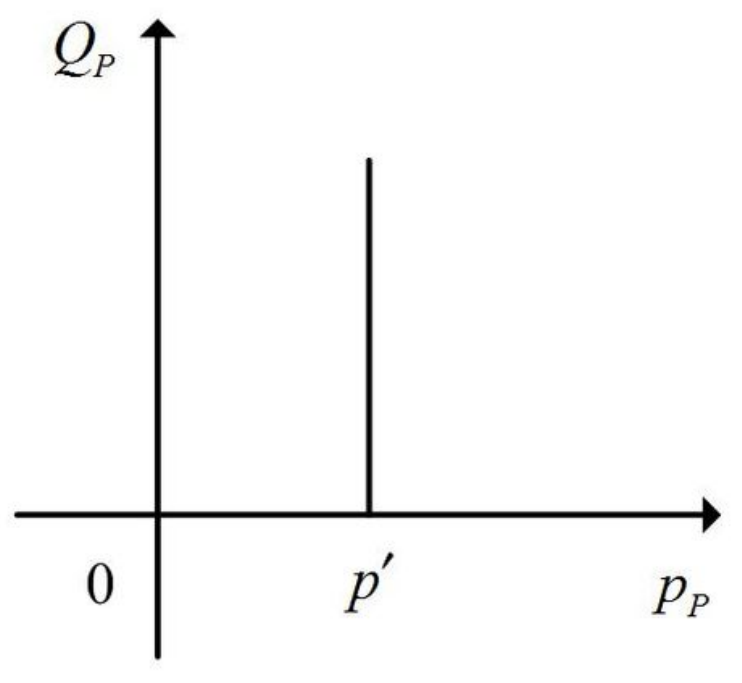

(d) Demand of PT

Figure 2

Demand functions of the two subsystem 
Station A - - - - - - - - $\rightarrow$ Station C

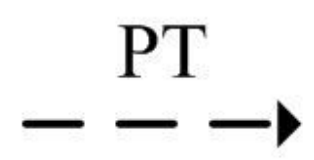

Figure 3

Customer's travel process for Scenario 1.
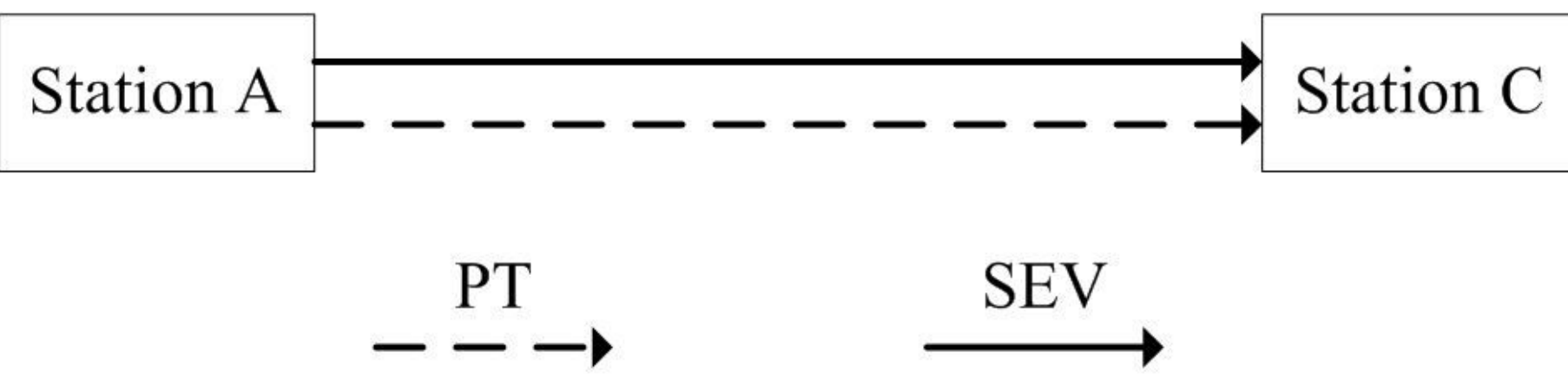

Figure 4

Customer travel process for Scenario 2.

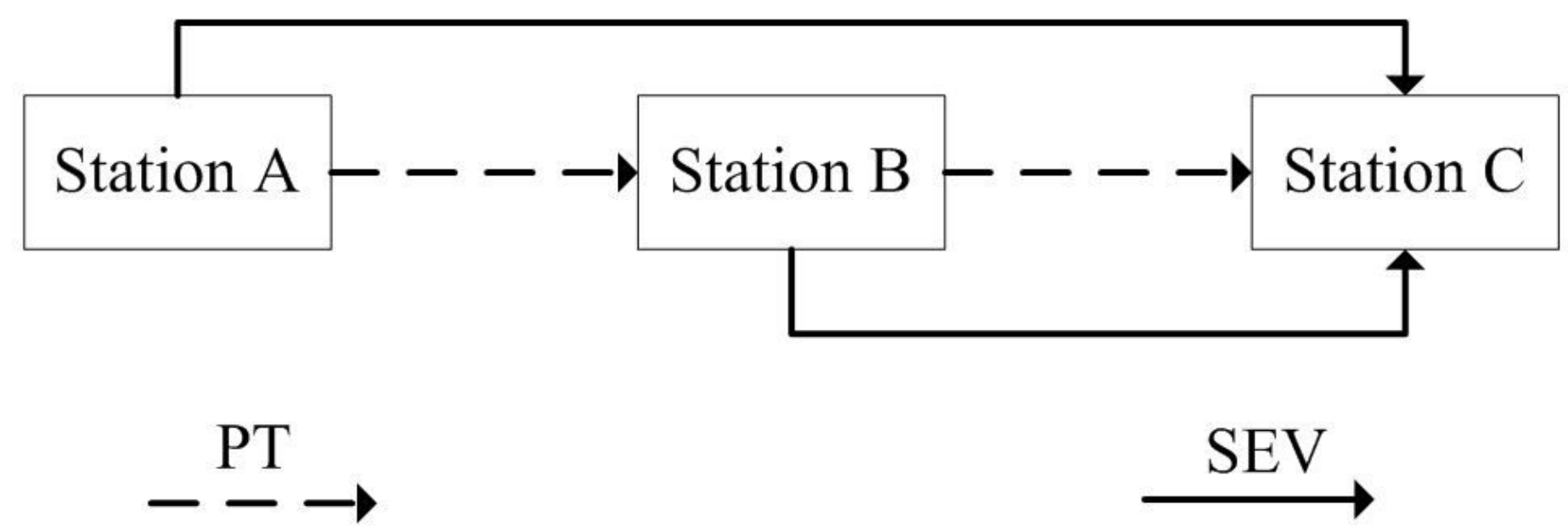

Figure 5

Customer travel process for Scenario 3. 

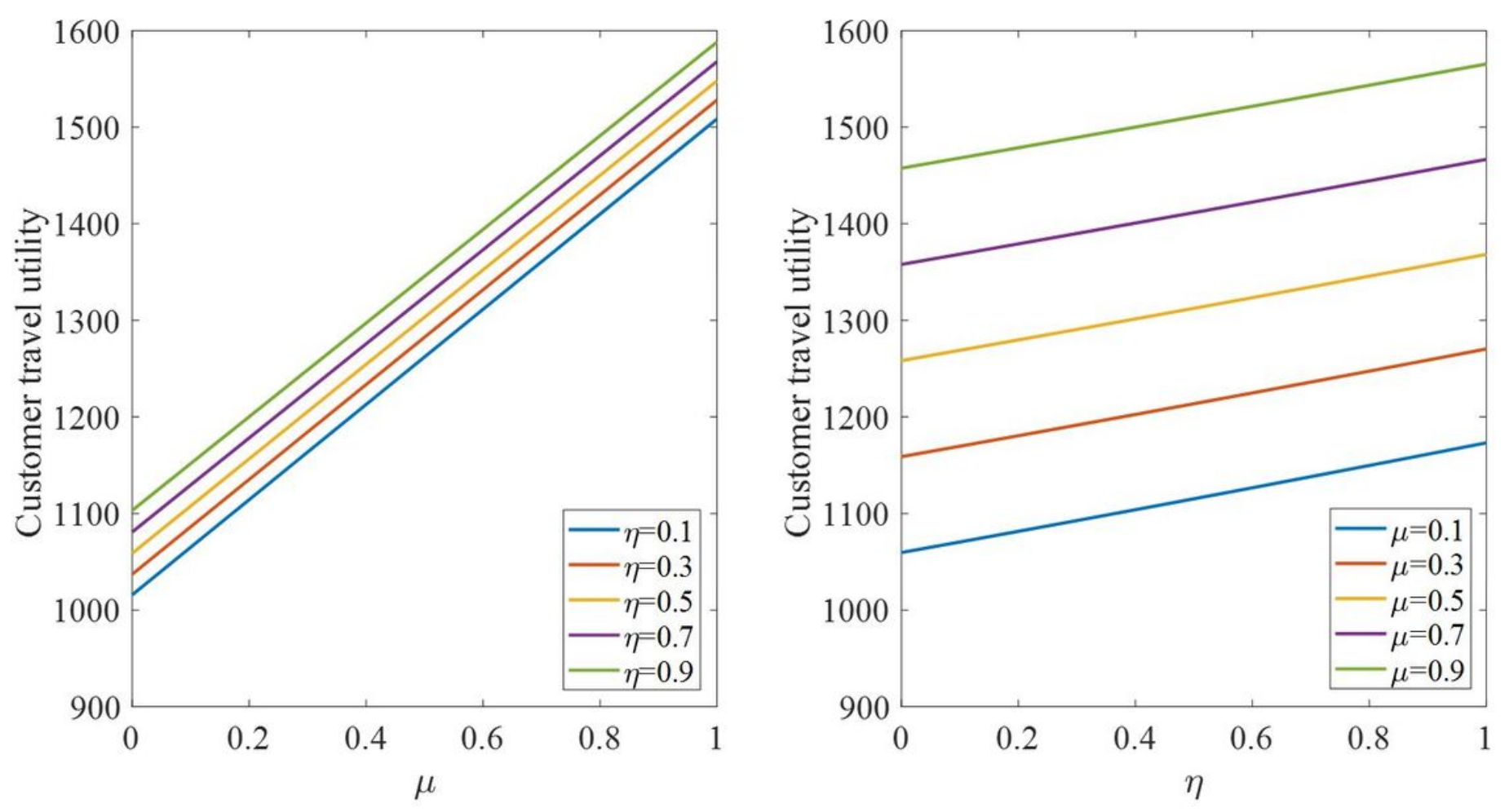

Figure 6

Sensitivity analysis under different values of $\mu$ and $\eta$.
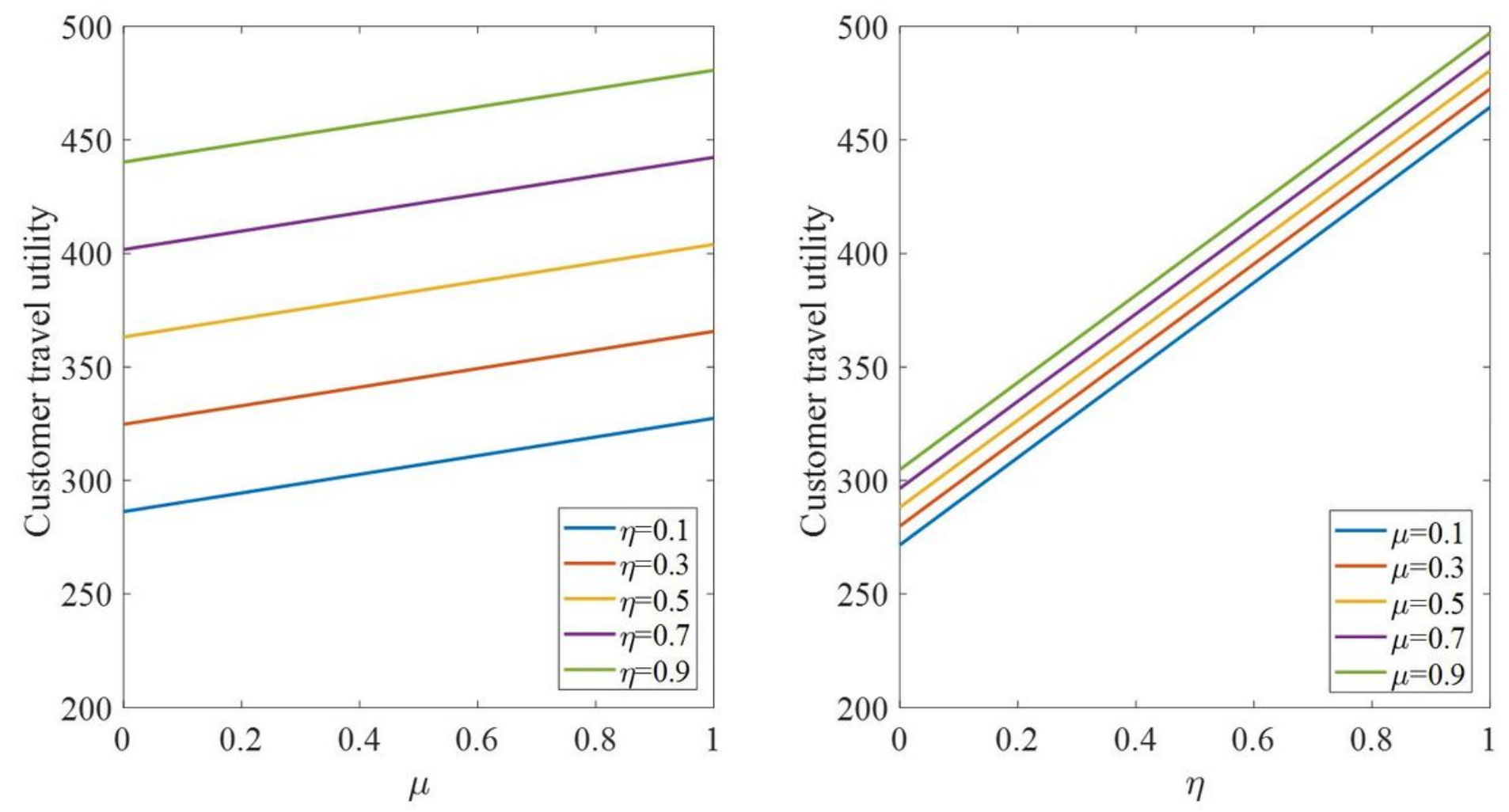

Figure 7 
Contrast experiment under different values of $\mu$ and $\eta$.
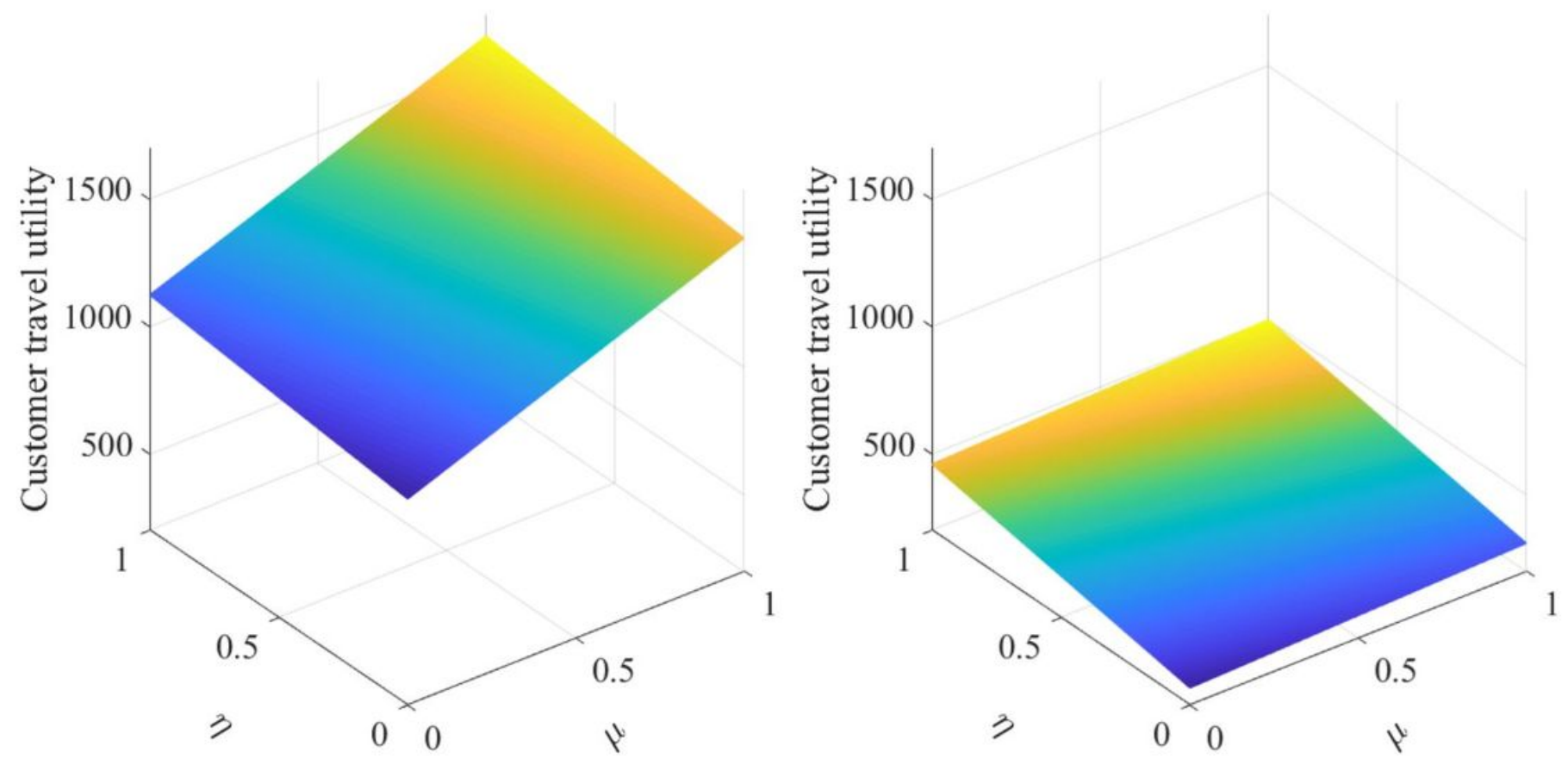

Figure 8

The impact of $\mu$ and $\eta$ on customer travel utility. 